\title{
O uso da cinoterapia associado a intervenção fisioterapêutica no tratamento de
}

\section{idosos com diagnóstico de depressão}

\author{
The use of cynotherapy associated with physiotherapeutic intervention in the treatment of elderly \\ with diagnosis of depression \\ El uso de cinoterapia asociada a intervención fisioterapéutica en el tratamiento de ancianos con \\ diagnóstico de depresión
}

\begin{abstract}
Resumo
À medida que o ser humano envelhece, os círculos sociais tendem a se reduzir, as pessoas costumam se isolar mais e podem desenvolver, muitas vezes, algum impacto nocivo em sua saúde emocional. Neste contexto, um animal de estimação pode ser uma grande fonte de alegria e fazer muita diferença ao longo do processo de envelhecimento. Observando essa condição, o presente estudo tem como objetivo analisar os efeitos da cinoterapia (Terapia Assistida por Animais) como intervenção cientificamente comprovada, associada no tratamento de idosos com diagnóstico de depressão. Trata-se de uma pesquisa bibliográfica e sistemática, englobando estudos datados do período de 2015 a 2019. Os resultados analisados demonstram que existem poucos estudos que tratam desse tema, e a maioria deles pede uma maior discussão dessa temática, corroborando para demonstrar o quão a terapia com animais é salutar e traz benefícios na saúde física e mental e qualidade de vida dos idosos .
\end{abstract}

Palavras-chave: Cães; Envelhecimento; Terapia.

\begin{abstract}
As human beings age, social circles tend to shrink, people tend to become more isolated and can often develop some harmful impact on their emotional health. In this context, a pet can be a great source of joy and make a lot of difference throughout the aging process. Observing this condition, the present study aims to analyze the effects of cynotherapy (Animal Assisted Therapy) as a scientifically proven intervention, associated with the treatment of elderly people diagnosed with depression. This is a bibliographic and systematic research, encompassing studies dating from 2015 to 2019 . The results analyzed show that there are few studies dealing with this topic, and most of them ask for a greater discussion of this theme, corroborating to demonstrate how much therapy with animals is healthy and brings benefits to the physical and mental health and quality of life of the elderly.
\end{abstract}

Keywords: Dogs; Aging; Therapy.

\section{Resumen}

A medida que los seres humanos envejecen, los círculos sociales tienden a reducirse, las personas tienden a aislarse más y, a menudo, pueden desarrollar algún impacto dañino en su salud emocional. En este contexto, una mascota puede ser una gran fuente de alegría y marcar una gran diferencia durante el proceso de envejecimiento. Observando esta condición, el presente estudio tiene como objetivo analizar los efectos de la cinoterapia (Terapia Asistida por Animales) como una intervención científicamente probada, asociada al tratamiento de personas mayores diagnosticadas con depresión. Se trata de una investigación bibliográfica y sistemática, que engloba estudios que datan del período 2015 a 2019. Los resultados analizados muestran que existen pocos estudios que abordan este tema, y la mayoría de ellos piden una mayor discusión sobre este tema, corroborando para demostrar cómo La terapia animal es saludable y aporta beneficios para la salud física y mental y la calidad de vida de las personas mayores.

Palabras clave: Perros; Envejecimiento; Terapia. 


\section{Introdução}

O envelhecimento é um processo natural presente em todos os indivíduos, sendo determinado como um estado progressivo em que ocorrem algumas modificações e consequências provenientes dessas alterações. No ser humano esse processo pode trazer muitas implicações negativas significativas que vão desde má qualidade de vida, dificuldades nas atividades de vida diária, comorbidades físicas, mortalidade prematura e déficits cognitivos. Em se tratando dos impactos relativos à condição psíquica, os transtornos depressivos são uma das doenças psiquiátricas mais comuns na população idosa (Santos et al., 2015).

De acordo com a Organização Mundial da Saúde (OMS), a prevalência de transtornos depressivos entre os idosos é de 10 a $20 \%$, e, infelizmente, a depressão entre os idosos é frequentemente considerada parte integrante da vida e é pouco reconhecida ou muitas vezes é subdiagnosticada (Varma, 2012).

Embora a incidência de depressão entre idosos seja semelhante à relatada na população adulta, a depressão em idosos está associada a maior risco de suicídio, hospitalização mais frequente, maior número de consultas nas agências de tratamento e sobrecarga familiar (Nóbrega et al., 2015).

Portanto, é muito importante reconhecer a depressão entre os idosos e administrar a mesma, fazendo o reconhecimento oportuno e o manejo adequado da depressão entre os idosos, buscando manter os níveis ideais de função e independência, redução da morbidade, redução da mortalidade por suicídio, desenvolvimento de doenças médicas e custos de tratamento (Varma, 2012).

Muitos estudos científicos vêm demonstrando que a inclusão de animais dentro do ambiente terapêutico já existe há muito tempo, desde o século XVII, e essa interação homem-animal dentro de processos terapêuticos traz o desenvolvimento de atividades em prol da melhoria da qualidade de vida, adotando, inclusive critérios específicos acompanhados por profissional da área da saúde em prol de aprimoramento de diversos aspectos da vida do paciente. Tal abordagem terapêutica é conhecida como conhecida como cinoterapia (Mandra et al., 2019).

A cinoterapia é uma modalidade de tratamento que utiliza o cão como facilitador para a realização de atividades recreativas promovendo melhora na saúde física e no aumento do estímulo para prática de exercícios físicos, ativando hormônios e neurotransmissores, como a dopamina, que agem de forma crucial amenizando sintomas depressivos (Silva, 2011).

No Brasil não existem muitos estudos que tratam desse tipo de tratamento, e os existentes estão voltados ao tratamento de crianças com transtornos de desenvolvimento, tais como o autismo, ou em pacientes que requerem alguma reabilitação física. Mas, nota-se que poucos estudos tratam sobre idosos e isso demonstra a importância e grande necessidade de fomento de novos estudos a respeito (Santos; Silva, 2016).

Uma outra forma de se referir ao recurso terapêutico com animais para afirmação de melhores condições de saúde é o que conhecemos como TAA (Animal Assisted Therapy) que é considerada uma intervenção realizada por profissional da área da saúde para alcançar objetivos claros e dirigidos em prol da melhoria do estado geral de saúde do idoso, demonstrando o quão essa terapêutica pode proporcionar uma melhora na saúde do idoso, com alta eficiência terapêutica, com benefícios biopsicossociais (Kobayashi et al., 2009), fazendo com que a pessoa que tenha contato com o animal tenha melhora no seu quadro geral de saúde e melhora do humor.

Assim, esse estudo enfrenta a seguinte problemática de pesquisa: a cinoterapia pode ajudar na melhoria da qualidade de vida e regressão dos quadros de depressão em idosos? Para responder a tal questionamento, elencou-se como objetivos da pesquisa: analisar os efeitos da cinoterapia associada no tratamento de idosos com diagnóstico de depressão, e especificamente busca tratar sobre a depressão na velhice e suas nuances, identificar a TAA como forma terapêutica e destacar os seus benefícios e melhoria na qualidade de vida, para, por fim, correlacionar o uso da cinoterapia no tratamento de idosos 
institucionalizados com depressão.

\section{Metodologia}

Trata-se de um estudo do tipo revisão integrativa, por ser um método de trabalho que busca a análise de pesquisas que se mostram relevantes para o aperfeiçoamento do conhecimento teórico e prático, na possibilidade da síntese do estado do conhecimento de um determinado assunto. Este método de pesquisa diferencia-se da revisão narrativa por permitir uma síntese de múltiplos estudos publicados e possibilitar conclusões gerais acerca de uma área de estudo específica (Minayo, 2008).

O estudo foi desenvolvido nos meses de setembro e outubro de 2021, a partir do levantamento de artigos científicos realizado nas bases de dados da Biblioteca Virtual em Saúde (BVS-BIREME): Scientific Electronic Library Online (SCIELO), e outras fontes com artigos científicos encontrados na base do Google acadêmico, utilizando os seguintes descritores: "Cinoterapia", "Depressão" e "Tratamento". Como critérios de inclusão inseriu-se estudos em inglês e português datados de 2010 a 2019 que tratam sobre o tema direta ou indiretamente, e como critérios de exclusão tem-se estudos mais antigos e artigos que não esteja incluído na integra.

Para selecionar as produções científicas, os critérios de inclusão foram artigos completos acerca da temática disponíveis online; todas as categorias de artigo (original, revisão de literatura, reflexão, atualização, relato de caso etc.); em língua portuguesa e inglesa. Os critérios de exclusão foram: monografias, dissertações, teses, artigos incompletos ou não disponíveis online e publicados em outros idiomas. Também foram excluídos artigos que não possuíam relação com a questão norteadora do estudo.

Os dados foram apresentados em forma de tabela, feita uma revisão, abordando os estudos, autor e ano e seus objetivos e considerações. Os dados foram analisados através da leitura dos estudos e análise de conteúdo, analisando documentos relacionados a temática e com isso será feito o fichamento e posterior escrita sobre o tema e discussão da temática abordada que discutirá os estudos elencados na tabela.

Vale salientar que o trabalho respeitou os aspectos éticos da lei de direitos autorais assegurando os direitos e deveres no que tange a comunidade científica.

\section{Resultados e Discussão}

A amostra deste estudo foi constituída por 10 (dez) artigos científicos que tratavam do tema Cinoterapia ou Terapia com animais, e relacionando ao tratamento de depressão ou ansiedade, e trouxessem informações variadas dos outros anteriormente selecionados, datados de 2010 a 2019 conforme pode ser visto na tabela abaixo (Tabela 1).

Tabela 1: Estudos científicos sobre da Cinoterapia associada no tratamento de Depressão. Vitória da Conquista, Bahia, Brasil, 2021.

\begin{tabular}{|c|c|c|c|c|c|}
\hline N. & $\begin{array}{c}\text { AUTORES } \\
\text { e ANO }\end{array}$ & TÍTULO & OBJETIVO & RESULTADO & CONCLUSÃO \\
\hline 1 & $\begin{array}{c}\text { Viau, et al. } \\
(2010)\end{array}$ & $\begin{array}{l}\text { Effect of service } \\
\text { dogs on salivary } \\
\text { cortisol secretion in } \\
\text { autistic children. }\end{array}$ & $\begin{array}{l}\text { Analisar os efeitos da interação } \\
\text { humana com cães e seus reflexos } \\
\text { no comportamento social. }\end{array}$ & $\begin{array}{l}\text { Sabemos que a interação humana com } \\
\text { cães, considerada um catalisador } \\
\text { social, resulta na diminuição dos } \\
\text { níveis de cortisol em adultos } \\
\text { saudáveis. }\end{array}$ & $\begin{array}{l}\text { Viu-se nos estudos da mostram que a } \\
\text { presença de cães dá suporte aos } \\
\text { potenciais benefícios do } \\
\text { comportamentais dos cães para } \\
\text { crianças com autismo. }\end{array}$ \\
\hline 2 & $\begin{array}{l}\text { Silva } \\
(2011)\end{array}$ & $\begin{array}{l}\text { Terapia Assistida por } \\
\text { Animais (Revisão de } \\
\text { Literatura). }\end{array}$ & $\begin{array}{l}\text { Estudar os principais meios } \\
\text { zooterápicos, como suas } \\
\text { indicações, e os benefícios } \\
\text { oferecidos citados na literatura } \\
\text { cientifica de uma forma clara e } \\
\text { objetiva. }\end{array}$ & $\begin{array}{l}\text { A zooterapia, método terapêutico } \\
\text { onde o animal é usado como parte } \\
\text { principal do tratamento, tem por } \\
\text { objetivo promover a saúde física, } \\
\text { social, emocional e/ou funções } \\
\text { cognitivas das pessoas. }\end{array}$ & $\begin{array}{l}\text { Ela parte do princípio de que o amor } \\
\text { e a amizade que podem surgir entre a } \\
\text { interação dos seres humanos e } \\
\text { animais geram inúmeros benefícios. }\end{array}$ \\
\hline
\end{tabular}




\begin{tabular}{|c|c|c|c|c|c|}
\hline 3 & $\begin{array}{l}\text { Moretti et } \\
\text { al. (2011) }\end{array}$ & $\begin{array}{l}\text { Pet therapy in elderly } \\
\text { patients with mental } \\
\text { illness. }\end{array}$ & $\begin{array}{l}\text { Avaliar os efeitos da pet terapia } \\
\text { no funcionamento cognitivo, } \\
\text { humor e qualidade de vida em } \\
\text { pacientes idosos com doenças } \\
\text { psiquiátricas. }\end{array}$ & $\begin{array}{l}\text { A terapia com animais ajuda no } \\
\text { tratamento de sintomas depressivos. }\end{array}$ & $\begin{array}{l}\text { A pet terapia mostrou-se eficiente na } \\
\text { melhora dos sintomas depressivos, } \\
\text { funcionamento cognitivo. }\end{array}$ \\
\hline 4 & $\begin{array}{l}\text { Reed, et al. } \\
\text { (2012) }\end{array}$ & $\begin{array}{l}\text { Curadores naturais: } \\
\text { uma revisão da } \\
\text { terapia e atividades } \\
\text { assistidas por } \\
\text { animais como } \\
\text { tratamento } \\
\text { complementar de } \\
\text { doenças crônicas }\end{array}$ & $\begin{array}{c}\text { Analisar as influências da } \\
\text { interação com cães no } \\
\text { comportamento com terapia e } \\
\text { atividades assistidas por animais } \\
\text { como tratamento complementar } \\
\text { de doenças crônicas. }\end{array}$ & $\begin{array}{l}\text { A pesquisa sugere que AAT/A é } \\
\text { eficaz para pacientes com diferentes } \\
\text { perfis. Descobriu-se que a interação } \\
\text { com cães incrementa comportamentos } \\
\text { positivos como aumento da } \\
\text { sensibilidade e atenção. }\end{array}$ & $\begin{array}{l}\text { Viu-se nos estudos da literatura sobre } \\
\text { o uso de terapia e atividades } \\
\text { assistidas por animais (AAT/A) que } \\
\text { essa ajuda até no combate a dor, e } \\
\text { melhora a qualidade de vida dos } \\
\text { pacientes. }\end{array}$ \\
\hline 5 & $\begin{array}{l}\text { Santos e } \\
\text { Silva } \\
(2016)\end{array}$ & $\begin{array}{c}\text { Os projetos de } \\
\text { terapia assistida por } \\
\text { animais no estado de } \\
\text { São Paulo. }\end{array}$ & $\begin{array}{l}\text { Identificar os projetos de TAA } \\
\text { existentes no estado de São Paulo } \\
\text { e caracterizá-los em relação ao } \\
\text { público alvo atendido, aos } \\
\text { coterapeutas utilizados e às } \\
\text { propostas apresentadas. }\end{array}$ & $\begin{array}{l}\text { A Terapia Assistida por Animais } \\
\text { (TAA) é uma intervenção planejada e } \\
\text { dirigida por profissionais que se } \\
\text { utilizam de animais como co- } \\
\text { terapeutas, os quais são parte } \\
\text { integrante do tratamento. }\end{array}$ & $\begin{array}{l}\text { Por meio desta pesquisa observamos } \\
\text { a necessidade e a importância da } \\
\text { terapia assistida por animais ser mais } \\
\text { dinamizada para que os seus } \\
\text { benefícios alcancem e auxiliem os } \\
\text { pacientes que sofrem em decorrência } \\
\text { de sua condição física e/ou psíquica. }\end{array}$ \\
\hline 6 & $\begin{array}{l}\text { Branson et } \\
\text { al. (2017). }\end{array}$ & $\begin{array}{l}\text { Depression, } \\
\text { loneliness, and pet } \\
\text { attachment in } \\
\text { homebound older } \\
\text { adult cat and dog } \\
\text { owners. }\end{array}$ & $\begin{array}{l}\text { Analisar a depressão, solidão e } \\
\text { apego a animais de estimação em } \\
\text { donos de cães e gatos idosos. }\end{array}$ & $\begin{array}{l}\text { A depressão é menor em pacientes } \\
\text { que tem animais de estimação, pois a } \\
\text { interação com animais serve como } \\
\text { terapia preventiva. }\end{array}$ & $\begin{array}{l}\text { Além disso, o contato com um } \\
\text { animal também fornece uma } \\
\text { oportunidade de interação social. }\end{array}$ \\
\hline 7 & $\begin{array}{l}\text { Dall, et al. } \\
\text { (2017). }\end{array}$ & $\begin{array}{l}\text { The influence of dog } \\
\text { ownership on } \\
\text { objective measures } \\
\text { of free-living } \\
\text { physical activity and } \\
\text { sedentary behavior } \\
\text { in community- } \\
\text { dwelling older } \\
\text { adults. }\end{array}$ & $\begin{array}{l}\text { Abordar essas questões usando } \\
\text { monitores para avaliar a } \\
\text { influência da posse de cães na } \\
\text { melhoria da saúde PA e SB em } \\
\text { um estudo longitudinal de adultos } \\
\text { idosos residentes na comunidade } \\
\text { com mobilidade independente. }\end{array}$ & $\begin{array}{c}\text { Há algumas evidências que sugerem } \\
\text { que a posse de um cão pode melhorar } \\
\text { a atividade física (AF) entre adultos } \\
\text { mais velhos }\end{array}$ & $\begin{array}{c}\text { A influência de cães na AF } \\
\text { encontrada neste estudo, indica que } \\
\text { pesquisas futuras sobre AF em } \\
\text { adultos mais velhos devem avaliar e } \\
\text { relatar tal situação, fomentando mais } \\
\text { estudos. }\end{array}$ \\
\hline 8 & $\begin{array}{l}\text { Paloski et } \\
\text { al. (2018) }\end{array}$ & $\begin{array}{l}\text { Efeitos da Terapia } \\
\text { Assistida por } \\
\text { Animais na } \\
\text { Qualidade de Vida } \\
\text { de Idosos: uma } \\
\text { Revisão Sistemática }\end{array}$ & $\begin{array}{l}\text { Investigar os efeitos da Terapia } \\
\text { Assistida por Animais (TAA) na } \\
\text { qualidade de vida em idosos por } \\
\text { meio de uma revisão sistemática. }\end{array}$ & $\begin{array}{l}\text { Os estudos destacam que a TAA } \\
\text { produz melhoria na qualidade de vida } \\
\text { dos idosos, e os instrumentos para } \\
\text { essa avaliação foram diversificados. }\end{array}$ & $\begin{array}{l}\text { Conclui-se que a TAA afeta } \\
\text { positivamente a qualidade de vida } \\
\text { dos idosos. }\end{array}$ \\
\hline 9 & $\begin{array}{l}\text { Mandra et } \\
\text { al. (2019) }\end{array}$ & $\begin{array}{l}\text { Terapia assistida por } \\
\text { animais: }\end{array}$ & $\begin{array}{c}\text { Verificar evidências sobre } \\
\text { aplicação da TAA na saúde } \\
\text { realizando revisão sistemática da } \\
\text { literatura. }\end{array}$ & $\begin{array}{l}\text { Os estudos clínicos prevaleceram que } \\
\text { a TAA foi usada preferencialmente } \\
\text { para reabilitação }\end{array}$ & $\begin{array}{c}\text { Há evidências científicas sobre o uso } \\
\text { da TAA publicadas no período } \\
\text { estudado, no Brasil e no mundo. }\end{array}$ \\
\hline 10 & $\begin{array}{l}\text { Cheryl et } \\
\text { al. (2019) }\end{array}$ & $\begin{array}{l}\text { Depression, and } \\
\text { Physical Activity in } \\
\text { Older Adults: The } \\
\text { Therapeutic Role of } \\
\text { Human-Animal } \\
\text { Interactions. }\end{array}$ & $\begin{array}{c}\text { Este artigo examina a literatura } \\
\text { existente sobre interações } \\
\text { humano-animal (HAIs) na vida } \\
\text { de adultos mais velhos em relação } \\
\text { a conceitos como solidão, } \\
\text { depressão e atividade física. }\end{array}$ & $\begin{array}{l}\text { Com o aumento previsto de adultos } \\
\text { mais velhos em todo o mundo, a } \\
\text { prevalência de solidão, depressão e } \\
\text { inatividade física também pode piorar } \\
\text { com o tempo se não houver } \\
\text { supervisão. }\end{array}$ & $\begin{array}{l}\text { Os idosos estão sujeitos a mudanças } \\
\text { psicológicas e físicas à medida que } \\
\text { envelhecem, e efetuar intervenções } \\
\text { para prevenir ou diminuir os desafios } \\
\text { psicológicos e físicos que os idosos } \\
\text { enfrentam é crucial. }\end{array}$ \\
\hline
\end{tabular}

Fonte: Própria pesquisa (2021).

Os estudos analisados demonstraram muitas vantagens de usar animais como apoio em terapia e a aplicação da TAA em idosos como uma forma de melhorar problemas de saúde mental. Notou-se nos estudos de Viau et al. (2010) e Silva (2011) destacam a interação de animais com seres humanos como elementar para efetivar uma interação e influindo no comportamento social.

Como demonstrado em um estudo realizado por Mandra et al. (2019), os cães são conhecidos por fazer uma diferença positiva para a saúde fisiológica dos humanos. Eles podem ser classificados como preventivos em problemas de saúde, facilitadores para a recuperação e desempenham um papel na saúde psicológica de humanos, reduzindo os níveis de estresse, ansiedade, depressão e solidão. E por isso, estudos como os de Moretti et al. (2011) e Branson et al. (2017) aduzem que os cães 
têm sido usados para fins terapêuticos em vários ambientes, com pessoas de todas as idades, sendo a Terapia Assistida por Animais um uso de cães em psicoterapia, melhorando sintomas de depressão e elevando a qualidade de vida.

Viau, et al. (2010) destacou que como pessoas com mais de 65 anos a terapia com animais é considerada um catalisador social, resulta na diminuição dos níveis de cortisol em e os idosos, o que foi corroborado por estudo de Branson et al. (2017), que geralmente sentem-se mais deprimidos, sozinhos quando tem seus próprios animais de estimação e contato com animal ele tem uma oportunidade de interação social e pode se exercitar com atividades assistidas por animais e esses desempenham um papel na redução do estresse, além de ter uma influência positiva no físico e saúde mental.

De acordo com Silva (2011) a interação animal pode melhorar os sintomas depressivos e diminuir significativamente pressão sanguínea, trazendo efeito positivo sobre os sintomas depressivos e qualidade de vida de idosos institucionalizados.

Reed et al. (2012) destaca em seu estudos que quando verifica-se o efeito da visitação assistida por animais em instalações de cuidados de longa duração, estudos relataram mudanças significativas nos escores de depressão do grupo de tratamento após quatro semanas de visitas com um cão guia, notando diminuição significativa na depressão, fadiga, tensão e confusão nos grupos de residentes e cães visitantes.

Moretti et al. (2011) relataram efeitos positivos da terapia com animais na saúde das pessoas, trazendo sensação de relaxamento e a redução do estresse, ansiedade e depressão, trazendo benefícios pessoais, e permitem que as pessoas interajam e se socializem e o toque de animais aumenta o comportamento social dos residentes em cuidados de longa duração.

Nota-se o efeito de um cão na depressão e melhoria nos níveis de ansiedade dos idosos. Reed, et al. (2012) aduz a melhora em quadros de algia e dor, e descobriu-se que a interação com cães incrementa comportamentos positivos como aumento da sensibilidade e atenção.

Santos e Silva (2016) também destacam a terapia com animais como uma técnica de tratamento com grande efeito positivo sobre o bem-estar psicológico dos residentes em instituições de longa permanência. Salientando que são necessários mais estudos com um grupo maior de participantes para ter melhor a representação da situação em estudo, fomentando pesquisas futuras a respeito desse tema.

Paloski et al. (2018) em consonância com os estudos acima, concluiu que a utilização da TAA gera efeitos positivos sobre a qualidade de vida de idosos, trazendo excelentes objetivos terapêuticos, destacando a necessidade de estudos futuros, sugerindo averiguar se a melhoria na qualidade de vida dos idosos se dá pelo contato com o cão, ou advém da interação com o terapeuta e o cão ou só com o terapeuta.

Branson et al. (2017) indica para os idosos e podem ter de escolher um cão para criar, pois o contato com o animal ameniza sintomas depressivos e reduz a ansiedade, e a depressão é menor em pacientes que tem animais de estimação, salientando a interação com animais como meio de terapia preventiva.

Dall, et al. (2017), por fim, acresce que o contato com cães pode melhorar até a capacidade funcional e atividade física de idosos, pois a influência de cães em atividades e interação de idosos é positiva e salutar, melhorando a saúde física e mental das pessoas eu interagem com os animais. Como benefícios diretos tem-se o fomento de alternativas efetivas e alternativas no combate a depressão em idosos.

\section{Conclusão}

Ao final da exposição deste presente estudo, notamos a importância de tratar da relação homem-animal em uma coesão que auxilie o ser humano a melhorar a sua saúde, principalmente a saúde mental, dentro de um processo de tratamento e relação com animais que melhore a qualidade de vida e bem estar.

Essa pesquisa apresentou-se sob a justificativa de tratar sobre um tema pouco abordado nos estudos científicos no Brasil, fazendo uma abordagem na perspectiva da importância de demonstrar como a acompanhamento assistencial através da 
cinoterapia serve na melhora da prevalência do quadro depressivo em idosos e é muito importante de modo a analisar os fatores relevantes que desencadeiam o aparecimento da doença e com isso enfatizar a importância de tratamento.

Assim, esse estudo serviu para contribuir cientificamente com o tema abordado e demonstrar aos profissionais de saúde a importância do acompanhamento e do desenvolvimento de ações desse tipo em prol do cuidado de idosos que apresentam quadros depressivos, podendo também ter influência direta ou indiretamente na construção de novos conhecimentos que possam ser somados à prática da melhora da qualidade de vida deles, servindo inclusive como modelo de terapia preventiva.

\section{Referências}

Branson, S. M., Boss, L., Cron, S., \& Turner, C. (2017) Depression, loneliness, and pet attachment in homebound older adult cat and dog owners. Journal of Mind and Medical Sciences, 4 (2),38-48.

Cheryl, A. Krause, P. Elise, E. Gulick, B. \& Basin, B. (2019) Depression, and Physical Activity in Older Adults: The Therapeutic Role of Human-Animal. Interactions, Anthrozoös, 32 (2), 239-254.

Dall, P. M., Ellis, S. L. H., Ellis, B. M., Grant, M., Colyer, A., Gee, N. R. \& Mills D. S. (2017). The influence of dog ownership on objective measures of freeliving physical activity and sedentary behavior in community-dwelling older adults: A longitudinal case-controlled study. BMC Public Health, 17 (3), 496.

Kobayashi, C. T., Ushiyama, S. T., Fakin, F. T., Robles, R. A. M., Carneiro, I. A., \& Carmagnani. M. I. S. (2009) Desenvolvimento e Implantação de Terapia Assistida por Animais em Hospital Universitário. Revista Brasileira Enfermagem, 62 (4), 632-6.

Mandra, P. P., et al. (2019) Terapia assistida por animais: revisão sistemática da literatura. CoDAS, 31 (3).

Minayo, M. C. S. (2008). O Desafio do Conhecimento: Pesquisa Qualitativa. (6a ed.), Hucitc.

Moretti, F., De Ronchi, D., Bernabei, V., Marchetti, L., Ferrari, B., Forlani, C. Negretti F., Sacchetti, L., \& Atti, A.R. (2011). Pet therapy in elderly patients with mental illness. Psychogeriatrics, 11(2), 125-129.

Nóbrega, I. R. A. P., Leal, M. C. C., Marques, A. P. O., \& Vieira, J. C. M. (2015). Fatores associados à depressão em idosos institucionalizados: revisão integrativa. Saúde Debate, Rio de Janeiro, 39 (105), 536-550.

Paloski, L. H., et al., (2018). Efeitos da Terapia Assistida por Animais na Qualidade de Vida de Idosos: uma Revisão Sistemática. Contextos Clínicos, 11 (2).

Pedrelli, P., Shapero, B., Archibald, A., \& Dale, C. (2016). Alcohol use and depression during adolescenceand young adulthood: a summary and interpretation of mixed findings. Curr Addict Rep, 3 (1) 91-97.

Reed, R., Ferrer, L., \& Villegas, N. (2012). Curadores naturais: uma revisão da terapia e atividades assistidas por animais como tratamento complementar de doenças crônicas. Rev. Latino-Am. Enfermagem, 20 (3).

Santos, A. R. O., \& Silva, C. de J. (2016) Os projetos de terapia assistida por animais no estado de São Paulo. Rev. SBPH, 19 (1), $133-146$.

Santos C, Ribeiro A, Rosa C, \& Ribeiro R. (2015). Depressão, déficit cognitivo e fatores associados à desnutrição em idosos com câncer. Ciênc Saúde Coletiva, 20 (3). 751-60.

Silva, J. M. (2011). Terapia Assistida por Animais (Revisão de Literatura). Tese de Monografia, Universidade Federal de Campina Grande- UFCG, Patos, MG.

Varma, G. S. (2012). Depression in the Elderly: Clinical Features and Risk Factors. Aging Dis, 3 (6), 465-471.

Viau, R., Arsenault-Lapierre, G., Fecteau, S., Champagne, N., Walker, C., \& Lupien, S. (2010). Effect of service dogs on salivary cortisol secretion in autistic children. 35 (2), 1187-93. 